\title{
The Sequence Project of the Control Plan of Reliability of the Weibull Model Distribution
}

\author{
Joanna Grubicka \\ Pomeranian Academy Slupsk, Poland \\ narl@poczta.onet.pl
}

(Received: 26 April 2010; revised: 16 June; accepted: 17 June 2010; published online: 27 September 2010)

\begin{abstract}
It is no exaggeration to say that the PN-IEC 61124 standard is inapplicable in many branches e.g. electronic industry. It is because PN-IEC 61124 assumes failure rate to be constant in time. This paper rejects this assumption and presents mathematical foundation of an alternative of PN-IEC 61124 applicable in electronic industry.
\end{abstract}

Key words: reliability test, Weibull distribution, Laplace transforms, renewal process

\section{INTRODUCTION}

It is no exaggeration to say that the IEC 6140 standard is inapplicable in many branchesof electronic industry. It is because PN-IEC 61124 assumes failure rate to be constant in time that is an obvious relict of the past. Willy-nilly, producers are forced to applyPN-IEC 61124 to their products being not aware of consequences expressed in terms of departures of OC curves from those presented in the standard.

This paper presents mathematical foundation of an alternative to PN-IEC 61124 applicable to electronic components and parts as well as a variety of other products that fail mostly due to early failures.

\section{MATHEMATICAL BASIS OF SEQUENTIAL TESTING}

Let us state two hypotheses related to reliability of the item of interest:

$H_{0}$ : The item is of satisfactory reliability. Its lifetime follows the Weibull model [5]

$$
f_{w}(t, a, b)=\frac{b}{a}\left(\frac{t}{a}\right)^{b-1} e^{-\left(\frac{t}{a}\right)^{b}},
$$

with parameter values $a=a_{0}, b=b_{0}, a>0, b>0, t \geq 0$.
$H_{1}$ : The item is of unsatisfactory reliability. Its lifetime follows the Weibull model (1) with parameter values $a=a_{1}, b=b_{1}$, usually $a_{1}<a_{0}, b_{1}<b_{0}$.

In a sequential test the decisions can be the following: either to accept $H_{0}$, or reject $H_{0}$ and thereby accept $H_{1}$, or, last but not least, delay the decision and continue the test.

We must keep in mind that the probability of performing exact $m$ renewals on $N$ laboratory positions observed in time $t$ from the beginning of the research follows the Poisson distribution:

$$
P(m, t / a, b)=\frac{[N \cdot H(t / a, b)]^{m}}{m !} \cdot \exp [-N \cdot H(t / a, b)]
$$

where: $m \in(0, \infty), \quad N=1,2, \ldots, H(t / a, b)$ is the renewal function provided that interarrival times follow the Weibull model (1). Derivation of the renewal function is presented in the next section.

To derive a decision rule we define the likelihood ratio

$$
L(t, m)=\frac{P_{1}\left(t, m / a_{1}, b_{1}\right)}{P_{0}\left(t, m / a_{0}, b_{0}\right)} .
$$

When $L(t, m) \ll<1$ it seems reasonable to accept $H_{0}$ and reject when $L(t, m)>>1$.

Combining (2) and (3) and transforming and simplifying the expression we get 


$$
L(t, m)=\left[\frac{H_{1}(t)}{H_{0}(t)}\right]^{m} \exp \left\{N \cdot\left[H_{0}(t)-H_{1}(t)\right]\right\},
$$

where:

$$
\begin{aligned}
& H_{1}(t)=H\left(t / a_{1}, b_{1}\right), \\
& H_{0}(t)=H\left(t / a_{0}, b_{0}\right) .
\end{aligned}
$$

A rule for making a decision is formulated as follows Accept $H_{0}$, if

$$
L \leq \frac{\beta}{1-\alpha}
$$

Reject $H_{0}$ and accept $H_{1}$, if

$$
L \geq \frac{1-\beta}{\alpha}
$$

Continue the test, if

$$
\frac{\beta}{1-\alpha}<L<\frac{1-\beta}{\alpha}
$$

where $\alpha, \beta$ are producer's and consumer's risks, respectively, $\alpha, \beta \in(0,1)$.

In practice, since these values $\alpha, \beta$ are usually smaller than 0,2 this particular choice of values $\beta /(1-\alpha)$, $(1-\beta) / \alpha$, gives real values close to reality.

Introducing the logarithmic likelihood ratio we would simplify further calculation. Namely,

$$
\begin{gathered}
l(t, m)=\ln L(t, m)=m \cdot \ln \left[\frac{H_{1}(t)}{H_{0}(t)}\right]+ \\
+N \cdot\left[H_{0}(t)-H_{1}(t)\right]=m \cdot \Omega(t)+N \cdot \Theta(t) .
\end{gathered}
$$

Definitions of auxiliary functions

$$
\begin{aligned}
& \Omega(t)=\ln \left[\frac{H_{1}(t)}{H_{0}(t)}\right], \\
& \Theta(t)=H_{0}(t)-H_{1}(t) .
\end{aligned}
$$

The test has to be continued while

$$
\frac{B-N \cdot \Theta(t)}{\Omega(t)}<m<\frac{A-N \cdot \Theta(t)}{\Omega(t)},
$$

where:

$$
B=\ln \frac{\beta}{1-\alpha}, \quad A=\ln \frac{1-\beta}{\alpha},
$$

otherwise the test is stopped [1].

\section{DERIVATION OF THE RENEWAL FUNCTION}

The relation between renewal function $H(t)$ and the renewal density function $\Lambda(t)$ is expressed by the following formula:

$$
H(t)=\int_{0}^{t} \Lambda(u) d u
$$

The renewal density function $\Lambda(t)$ and the failure density function $f(t)$ are interrelated.

$$
\Lambda(t)=f(t)+\int_{0}^{t} \Lambda(\tau) \cdot f(t-\tau) d \tau .
$$

Applying the Laplace transform to both sides of (10) we get

$$
\Lambda(s)=f(s)+f(s) \cdot \Lambda(s)
$$

and after simple transformation [4]

$$
\Lambda(s)=\frac{f(s)}{1-f(s)} .
$$

Unfortunately, in case of the Weibull lifetime model this equation cannot be solved analytically. To obtain an approximate solution we employ the mixed exponential model

$$
\begin{gathered}
f_{S}(t)=\sum_{i=1}^{3} \omega_{i} \cdot \lambda_{i} \cdot e^{-\lambda_{i} \cdot t} \quad \sum_{i=1}^{3} \omega_{i}=1 \\
\lambda_{i}>0, i=1,2,3
\end{gathered}
$$

as a surrogate model to replace the original one.

The surrogate model has three desired features: simplicity, flexibility and transformability in Laplace sense. Fraction parameters $\omega_{i}$ and scale parameters $\lambda_{i}, \quad i=1,2,3$ are chosen in such a way that they make the surrogate model most similar to the original Weibull distribution.

The similarity measure especially developed by the authors [2] for this purpose is:

$$
\operatorname{SM}(\bar{\omega}, \bar{\lambda})=\int_{0}^{\infty} \min _{t}\left[f_{s}(t, \bar{\omega}, \bar{\lambda}), f_{w}(t, a, b)\right] d t,
$$

where $\bar{\omega}, \bar{\lambda}$ are vectors of fraction and scale parameters mentioned above.

Let $\bar{\omega}^{*}, \bar{\lambda}^{*}$ be vectors that maximize (13). The Laplace transform $L(s)$ corresponding to $(12)$ is 


$$
\begin{gathered}
f_{s}(s)=L\left[\sum_{i=1}^{3} \omega_{i}^{*} \cdot \lambda_{i}^{*} \cdot e^{-\lambda_{i} \cdot t}\right]= \\
=\sum_{i=1}^{3} \omega_{i}^{*} \cdot \lambda_{i}^{*} \cdot L\left[e^{-\lambda_{i} \cdot t}\right]=\sum_{i=1}^{3} \omega_{i}^{*} \cdot \lambda_{i}^{*} \cdot \frac{1}{s+\lambda_{i}^{*}} .
\end{gathered}
$$

Substituting (14) to (11b) we get

$$
\Lambda(s)=\frac{\sum_{i=1}^{3} \frac{\omega_{i}{ }^{*} \cdot \lambda_{i}{ }^{*}}{s+\lambda_{i}{ }^{*}}}{1-\sum_{i=1}^{3} \frac{\omega_{i}{ }^{*} \cdot \lambda_{i}{ }^{*}}{s+\lambda_{i}{ }^{*}}}
$$

after simple although arduous transformations we get

$$
\Lambda(s)=\frac{L_{2} s^{2}+L_{1} s+L_{0}}{M_{3} s^{3}+M_{2} s^{2}+M_{1} s+M_{0}},
$$

where:

$$
\begin{gathered}
L_{2}=\omega_{1}{ }^{*} \cdot \lambda_{1}{ }^{*}+\omega_{2}{ }^{*} \cdot \lambda_{2}{ }^{*}+\omega_{3}{ }^{*} \cdot \lambda_{3}{ }^{*}, \\
L_{1}=\omega_{1}{ }^{*} \cdot \lambda_{1}{ }^{*} \cdot\left(\lambda_{2}{ }^{*}+\lambda_{3}{ }^{*}\right)+\omega_{2}{ }^{*} \cdot \lambda_{2}{ }^{*} \cdot\left(\lambda_{1}{ }^{*}+\lambda_{3}{ }^{*}\right)+ \\
+\omega_{3}{ }^{*} \cdot \lambda_{3}{ }^{*} \cdot\left(\lambda_{1}{ }^{*}+\lambda_{2}{ }^{*}\right), \\
L_{0}=\lambda_{1}{ }^{*} \cdot \lambda_{2}{ }^{*} \cdot \lambda_{3}{ }^{*}, \\
M_{3}=1, \\
M_{2}=\lambda_{1}{ }^{*} \cdot\left(1-\omega_{1}{ }^{*}\right)+\lambda_{2}{ }^{*} \cdot\left(1-\omega_{2}{ }^{*}\right)+\lambda_{3}{ }^{*} \cdot\left(1-\omega_{3}{ }^{*}\right), \\
M_{1}=\lambda_{1}{ }^{*} \cdot \lambda_{2}{ }^{*} \cdot \omega_{3}{ }^{*}+\lambda_{1}{ }^{*} \cdot \lambda_{3}{ }^{*} \cdot \omega_{2}{ }^{*}+\lambda_{2}{ }^{*} \cdot \lambda_{3}{ }^{*} \cdot \omega_{1}{ }^{*}, \\
M_{0}=0 .
\end{gathered}
$$

Derivation of the renewal density function can be simplified when we convert (16) into part fraction

$$
\Lambda(s)=\frac{\Lambda_{0}}{s}+\frac{\Lambda_{1}}{s-s_{1}}+\frac{\Lambda_{2}}{s-s_{2}},
$$

where $s_{1}, s_{2}$ are poles of (16), namely

$$
s_{1,2}=\frac{\left(-M_{2} \pm \sqrt{M_{2}^{2}-4 M_{1}}\right)}{2}
$$

and

$$
\begin{gathered}
\Lambda_{0}=\frac{L_{0}}{s_{1} \cdot s_{2}}, \quad \Lambda_{1}=\frac{L_{2} s_{1}^{2}+L_{1} s_{1}+L_{0}}{s_{1}\left(s_{1}-s_{2}\right)}, \\
\Lambda_{2}=\frac{L_{2} s_{2}^{2}+L_{1} s_{2}+L_{0}}{s_{2}\left(s_{2}-s_{1}\right)}
\end{gathered}
$$

are corresponding residues [3].

Thus, we obtain renewal density function performing inverse Laplace transform $L^{-1}(s)$

$$
\begin{gathered}
\Lambda(t)=L^{-1}[\Lambda(s)]=L^{-1}\left[\frac{\Lambda_{0}}{s}\right]+L^{-1}\left[\frac{\Lambda_{1}}{s-s_{1}}\right]+ \\
+L^{-1}\left[\frac{\Lambda_{2}}{s-s_{2}}\right]=\Lambda_{0}+\Lambda_{1} e^{-\left|s_{1}\right| t}+\Lambda_{2} e^{-\left|s_{2}\right| t} .
\end{gathered}
$$

Finally, the renewal function we need in the sequential test takes the form

$$
H(t)=\int_{0}^{t} \Lambda(t) d t=\Lambda_{0} \cdot t+
$$

$$
\begin{gathered}
+\frac{\Lambda_{1}}{\left|s_{1}\right|} \cdot\left(1-e^{-\left|s_{1}\right| \cdot t}\right)+\frac{\Lambda_{2}}{\left|s_{2}\right|} \cdot\left(1-e^{-\left|s_{2}\right| \cdot t}\right)= \\
=H_{a} \cdot t+H_{b} \cdot\left(1-e^{-\left|s_{1}\right| \cdot t}\right)+H_{c} \cdot\left(1-e^{-\left|s_{2}\right| \cdot t}\right),
\end{gathered}
$$

where:

$$
H_{a}=\Lambda_{0}, \quad H_{b}=\frac{\Lambda_{1}}{\left|s_{1}\right|}, \quad H_{c}=\frac{\Lambda_{2}}{\left|s_{2}\right|}
$$

\section{EXAMPLE}

Table 1. Parameters of the lifetime models ascribed to items of satisfactory and unsatisfactory reliability

\begin{tabular}{c|c|c}
\hline \multirow{2}{*}{$\begin{array}{c}\text { Weibull } \\
\text { parameters }\end{array}$} & \multicolumn{2}{|c}{ Reliability } \\
\cline { 2 - 3 } & satisfactory & unsatisfactory \\
\hline$a$ & 3 & 1 \\
\hline$b$ & 0.9 & 0.5 \\
\hline
\end{tabular}

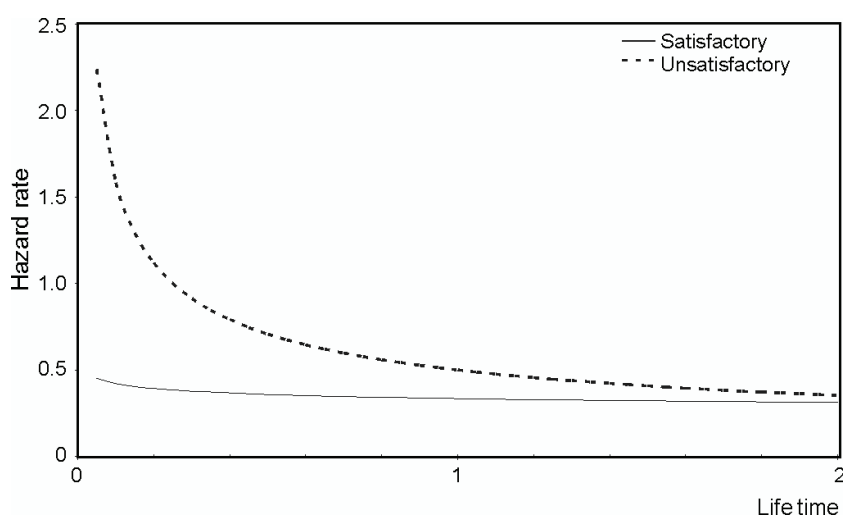

Fig. 1. Hazard rate functions ascribed to items of satisfactory and unsatisfactory reliability 


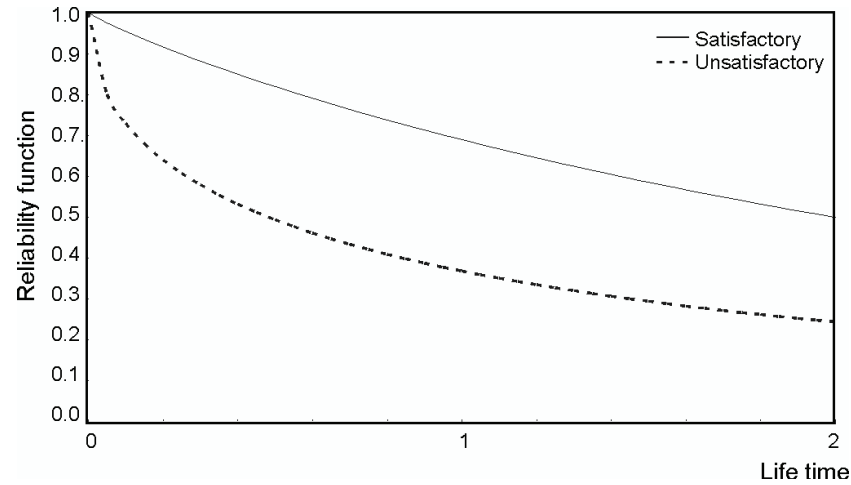

Fig. 2. Reliability functions ascribed to items of satisfactory and unsatisfactory reliability

Table 2. Parameters of the surrogate distribution

\begin{tabular}{c|c|c}
\hline \multirow{2}{*}{$\begin{array}{c}\text { Surrogate } \\
\text { parameters }\end{array}$} & \multicolumn{2}{|c}{ Reliability } \\
\cline { 2 - 3 } & satisfactory & unsatisfactory \\
\hline$\omega_{1}$ & 0.00648 & 0.18974 \\
\hline$\omega_{2}$ & 0.13509 & 0.41179 \\
\hline$\omega_{3}$ & 0.85843 & 0.39847 \\
\hline$\lambda_{1}$ & 30.02119 & 21.79711 \\
\hline$\lambda_{2}$ & 1.11207 & 1.80952 \\
\hline$\lambda_{3}$ & 0.28745 & 0.24915 \\
\hline
\end{tabular}

Table 3. Calculation parameters of the surrogate distribution (intermediate results)

\begin{tabular}{c|c|c}
\hline \multirow{2}{*}{$\begin{array}{c}\text { Polynomial } \\
\text { Coefficients }\end{array}$} & \multicolumn{2}{|c}{ Reliability } \\
\cline { 2 - 3 } & Satisfactory & Unsatisfactory \\
\hline$L_{2}$ & 0.591522551 & 4.980204693 \\
\hline$L_{1}$ & 12.50782126 & 27.28545122 \\
\hline$L_{0}$ & 9.596709336 & 9.827050661 \\
\hline$M_{3}$ & 1 & 1 \\
\hline$M_{2}$ & 30.82918745 & 18.87557531 \\
\hline$M_{1}$ & 29.82709909 & 18.03844713 \\
\hline$M_{0}$ & 0 & 0 \\
\hline
\end{tabular}

Table 4. Calculation parameters of the renewal function

\begin{tabular}{c|c|c}
\hline \multirow{2}{*}{$\begin{array}{c}\text { Renewal } \\
\text { parameters }\end{array}$} & \multicolumn{2}{|c}{ Reliability } \\
\cline { 2 - 3 }$s_{1}$ & -0.99992756 & -1.009656861 \\
\hline$s_{2}$ & -29.8292598 & -20.72677411 \\
\hline$\Lambda_{1}$ & 0.321744643 & 0.469588746 \\
\hline$\Lambda_{2}$ & 0.080436722 & 0.635188875 \\
\hline$\Lambda_{3}$ & 0.178555812 & 3.852529843 \\
\hline$H_{a}$ & 0.321744643 & 0.469588746 \\
\hline$H_{b}$ & 0.08044255 & 0.629113612 \\
\hline$H_{c}$ & 0.005985928 & 0.185872139 \\
\hline
\end{tabular}

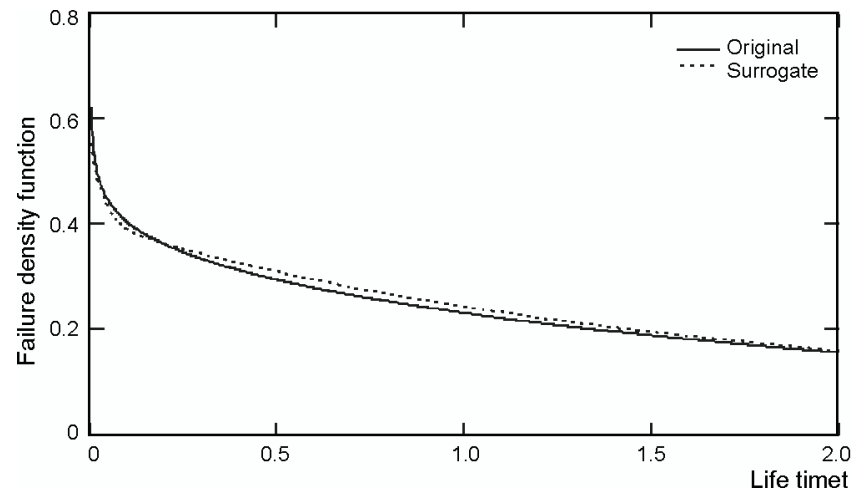

Fig. 3. The original and surrogate models in the case of unsatisfactory reliability. A comparison

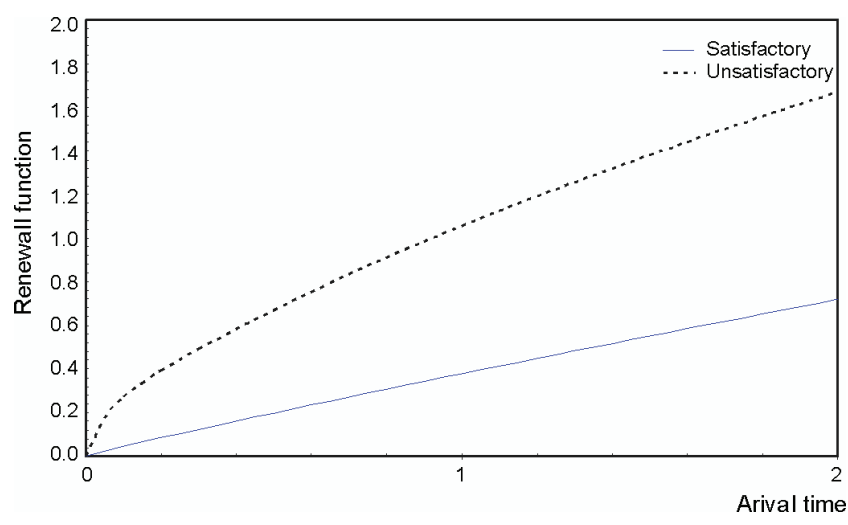

Fig. 4. Renewal functions ascribed to items of satisfactory and unsatisfactory reliability 


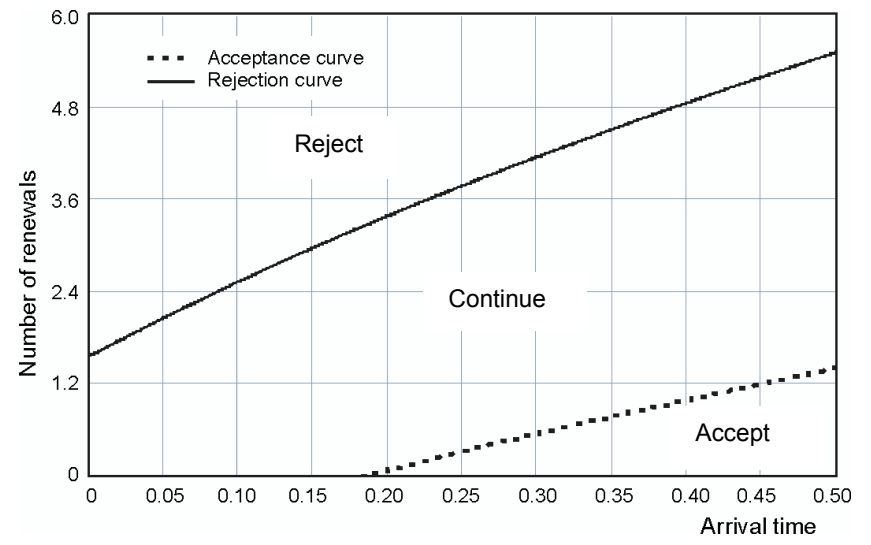

Fig. 5. A grid of the sequential compliance test

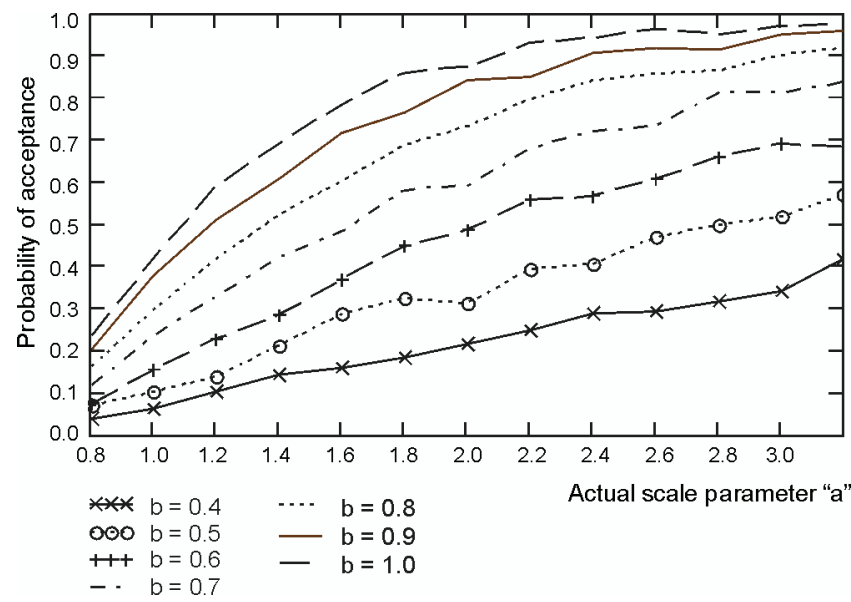

Fig. 6. Family of OC curves obtained with the Monte Carlo method

\section{CONCLUSION}

At first glance, the grid is similar to those of PN-IEC 61124 for constant failure rate. There is, however, a signiicant difference. Borders between "Reject", "Continue the test" and "Accept" regions are nonlinear. This is a direct consequence of nonlinearity of the renewal function. However, a decision making rule remains unchanged.

The theory presented in the article allows to design the control plans of reliability, which might be found aplicable in electronic industry.

\section{References}

[1] A. Drapella, Lifetime models and renewal processes. Numerical treatment with Mathcad. Pomeranian Pedagogical Academy, Słupsk, 72-73 (2002).

[2] A. Drapella, Metoda fuzji i korekcji danych niezawodnościowych. Raport z pracy w ramach Grant KBN System ekspertowy wspomagania analiz niezawodnościowych i probabilistycznych zespołów energetycznych. Politechnika Gdańska 1994.

[3] J. Grubicka, Wyznaczanie gęstości odnowy metodq rozkładu zasteppczego. Warszawa 2004 (PAN, praca doktorska).

[4] J. Grubicka, Wyznaczanie przybliżonej gęstości odnowy w przypadku weibullowskiego rozkładu czasu pracy do uszkodzenia metoda rozktadu zastępczego. (cz. II) SPM-F 25 (2002).

[5] N.L Johnson, S. Kotz, Distributions in statistics. Continuous univariate distributions. (2 vols.) Houghton Mifflin, Boston 1970.

[6] PN-IEC 61124:2003. Badanie nieuszkadzalności - Badania zgodności $w$ przypadku stałej intensywności uszkodzeń $i$ stałej intensywności strumienia uszkodzeń.

[7] PN-EN 61124:2006. Badanie nieuszkadzalności - Badania zgodności w przypadku statej intensywności uszkodzeń $i$ stałej intensywności strumienia uszkodzeń.

[8] PN-IEC 60605-6:2002. Badanie nieuszkadzalności urzadzeń. Część 6: Weryfikowanie hipotez o stałej intensywności uszkodzeń i statej intensywności strumienia uszkodzeń.

[9] PN-EN 61703:2005. Wyrażenia matematyczne dotyczace nieuszkadzalności, gotowości, obstugiwalności i zapewnienia środków obstugi.

[10] W. Weibull, Statistical distribution function of wide applicability. Journal Applied Mechanics 293-317 (1951).

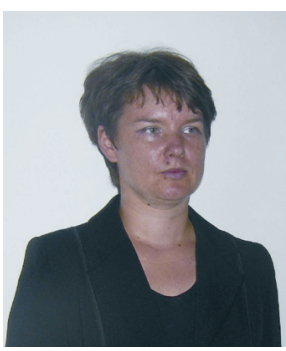

JoANNA GrUbiCKA graduated in Mathematics in 1997. Since then she has been working at the Institute of Mathematics at Pomeranian Academy in Słupsk. She received the $\mathrm{PhD}$ in reliability theory in 2005 from the Systems Research Institute of Polish Academy of Sciences in Warsaw. Her research interests concern reliability mathematics and computational methods in statistics. 